\title{
Long-Term Serological Response to 13-Valent Pneumococcal Conjugate Vaccine Versus 23-Valent Polysaccharide Vaccine in HIV-Infected Adults
}

\author{
Simone Belmonti - Barbara Rossetti - Sara Modica - Lorenzo Paglicci • \\ Alberto Borghetti - Arturo Ciccullo - Chiara Picarelli · Roberto Cauda • \\ Andrea De Luca · Francesca Montagnani · Francesca Lombardi (D)
}

Received: June 10, 2019 / Published online: July 30, 2019

(c) The Author(s) 2019

\section{ABSTRACT}

Introduction: Long-term

comparative

immunologic response to 13 -valent pneumococcal conjugate vaccine (PCV13) versus 23-valent polysaccharide vaccine (PPV23) among HIV-infected adults has not yet been investigated.

Methods: In this prospective pilot study, we quantified in HIV-positive adults serotype-

Enhanced Digital Features To view enhanced digital features for this article go to https://doi.org/10.6084/ m9.figshare.8937809.

Electronic supplementary material The online version of this article (https://doi.org/10.1007/s40121019-0256-z) contains supplementary material, which is available to authorized users.

S. Belmonti · A. Ciccullo · C. Picarelli · R. Cauda · F. Lombardi $(\bowtie)$

Istituto di Clinica Malattie Infettive, Università

Cattolica del Sacro Cuore, Rome, Italy

e-mail: francesca.lombardi@gmail.com

B. Rossetti - A. De Luca · F. Montagnani

Hospital Department of Specialized and Internal Medicine, University Division of Infectious Diseases, Viale Bracci 16, 53100 Siena, Italy

S. Modica - L. Paglicci - A. De Luca · F. Montagnani Department of Medical Biotechnologies, University of Siena, Viale Bracci 16, 53100 Siena, Italy

A. Borghetti · R. Cauda

Fondazione Policlinico Universitario A. Gemelli

IRCCS, UOC Malattie Infettive, Rome, Italy specific IgG concentrations of the 12 pneumococcal serotypes shared by both vaccines 5 years after vaccination with two doses of PCV13 8 weeks apart (group 1) or one dose of PPV23 (group 2) and compared them with those assessed prior to vaccination (BL) and after 1 year (T1). Comparison of immunogenicity was based on geometric mean concentration (GMC), proportion of individuals with $\geq$ twofold increase from BL in specific antibody concentration against $\geq 2$ serotypes and percentage of individuals with serotype-specific IgG $\geq 0.35 \mu \mathrm{g} / \mathrm{ml}, \geq 1 \mu \mathrm{g} / \mathrm{ml}$ and $\geq$ individual serotype-specific correlates of protection.

Results: We included 91 subjects (median $\mathrm{CD}^{+} 650$ cells $/ \mu \mathrm{l},>90 \%$ with HIV-RNA $<50$ copies $/ \mathrm{ml})$; patients in groups $1(n=42)$ and 2 $(n=49)$ were homogeneous for the main characteristics. GMCs were significantly higher in the PCV13 group than in the PPV23 group for serotype $19 \mathrm{~F}$ ( $p=0.003)$. Both vaccines revealed higher significant GMCs to most serotypes compared with BL, i.e., eight in group 1 vs. seven in group 2. With respect to T1, GMCs decreased significantly in the PCV13 group for eight vs. ten serotypes in the PPV23 group. More participants in the PCV13 group had $\geq 2$ increase from BL in antibody levels to $\geq 2$ serotypes compared with the PPV23 group (78.6\% vs. $59.2 \%, p=0.042)$. Overall, the percentage of subjects with serotype-specific IgG $\geq 0.35 \mu \mathrm{g} /$ $\mathrm{ml}, \geq 1 \mu \mathrm{g} / \mathrm{ml} \quad$ and $\geq$ individual serotype- 
specific correlates of protection was similar between groups.

Conclusion: In this study with HIV-positive adults with a favorable viro-immunologic profile, both vaccines were shown to achieve a long-term durable serologic response. We found minor differences in immunogenicity between the two vaccines, which favored PCV13 over PPV23 5 years after immunization.

Trial Registration: ClinicalTrials.gov identifier, NCT02123433.

Keywords: HIV; PCV13; Pneumococcal vaccination; PPV23; Serologic response; Streptococcus pneumoniae

\section{INTRODUCTION}

Despite the improved quality of life achieved by HIV-infected patients mainly due to current antiretroviral therapy efficacy and tolerability, HIV-infected people are at higher risk for Streptococcus pneumoniae infections than age-matched HIV-uninfected adults [1]; this risk may be due to HIV-related accelerated senescence of the immune repertoire, loss of memory cells and the relative dysregulation of the immune system $[2,3]$.

Thus, pneumococcal vaccination is mandatory for HIV-infected adults, but the optimal time schedule still needs to be clarified. Two vaccine formulations are currently available: 23-valent pneumococcal polysaccharide vaccine (PPV23) and 13-valent pneumococcal conjugate vaccine (PCV13), which replaced the previous 7-valent pneumococcal conjugate vaccine (PCV7). The magnitude and duration of responses to the pneumococcal polysaccharide vaccine may be limited by its nature, and data on its effectiveness in preventing pneumococcal disease are inconsistent. By contrast, the conjugated vaccine, which elicits a $\mathrm{T}$ cell-dependent response and memory $\mathrm{B}$ and $\mathrm{T}$ cells, has been shown to be effective against vaccine-type invasive pneumococcal disease in a randomized controlled trial of HIV-infected adults [4].

Therefore, current guidelines recommend that PCV13 be given as the initial vaccination for HIV-positive adults and that PPV23 be administered subsequently to broaden serotype coverage [5]. However, a benefit of PCV vs. PPV in terms of immunogenicity among HIV-positive adults has not been fully established [6-8].

Furthermore, there is a lack of data on the long-term immunogenicity of PCVs compared with PPV in this population. The characteristic of PCVs to elicit immunologic memory could hypothetically be an advantage in the long term, but this still has to be demonstrated in HIV-infected subjects.

Previous data showed that in HIV-positive adults who were receiving antiretroviral therapy, response to the pneumococcal polysaccharide vaccine decreased for most of the serotypes after 5 years [9]. In a similar setting, a different study showed that one or two doses of PCV7 ensured a durable serologic response throughout the 5 years of follow-up, but PPV23 was not used as a comparator vaccine in this study [10]. Overall, there is scanty evidence of persistent serologic response to immunization with PCV.

Thus, further investigations are mandatory, and the long-term comparative immunogenicity of protein-conjugated and free polysaccharide vaccines in HIV-positive adults needs to be addressed.

In a 1-year follow-up report, we previously showed that vaccination with the 13 -valent pneumococcal conjugate vaccine or with the 23-valent pneumococcal polysaccharide vaccine elicited comparable immunogenicity in a cohort of HIV-positive adults with a favorable viro-immunologic profile [11].

Here we aimed to compare the long-term (5year) serologic response in those subjects previously immunized with PCV13 or PPV23.

\section{METHODS}

In a pilot prospective study, we included pneumococcal vaccine-naïve HIV-infected outpatients who were matched for age (18-65 years) and $\mathrm{CD}^{+}$cell count $(\geq 200$ cells $/ \mu \mathrm{l})$; they received two doses of PCV13 8 weeks apart (group 1) or one dose of PPV23 (group 2). As described in the previous report, patients were enrolled in two parallel Infectious Diseases 
Clinical Centers in Central Italy (University Division of Infectious Diseases in Siena and Institute of Clinical Infectious Diseases, Catholic University of the Sacred Heart in Rome) [11].

All procedures performed in the study were in accordance with the 1964 Helsinki Declaration and its later amendments or comparable ethical standards. The present study was approved by the local Institutional Ethics Committees, Comitato Etico Fondazione Policlinico Universitario "Agostino Gemelli"Università Cattolica del Sacro Cuore and Comitato Etico Regione Toscana-Area vasta Sud Est, AOU Senese (protocol no. 13379/16 and protocol code PCV13HIV-BOOST-2014). Written informed consent was obtained from each participant.

Serotype-specific IgG concentration for the 12 pneumococcal serotypes shared by both vaccines was quantified by ELISA 5 years (T5) after the vaccination, as previously reported [12]. The antibody concentrations were logtransformed to approximate normal distributions and parametric methods were applied in the analysis. Results of IgG antibody levels were expressed as geometric mean concentrations (GMCs). The GMCs were obtained by taking the antilogarithm of the means of the log-transformed values. Corresponding two-sided 95\% confidence intervals (CIs) for the GMCs were constructed by back-transforming the 95\% CI for the mean of logarithmically transformed assay results computed using Student's $t$ distribution.

The primary end point of this report was to compare the long-term serologic response at $\mathrm{T} 5$ by assessing the specific IgG levels for the 12 serotypes between the two groups, using a linear regression adjusted for age, gender and CD4 cell count. The comparison of IgG levels for each serotype at $\mathrm{T} 5$ with respect to those assessed prior to vaccination (BL) and after 1 year (T1) intra-groups was performed by applying a generalized linear model (GLMM) by specifying correlation between repeated measures within subjects and adjusting using the Bonferroni method in the pairwise comparisons of time points. The GLMM was also adjusted for gender, age and CD4, which was considered a timevarying variable.
As the secondary end point, we compared the immunogenicity of the two vaccines by measuring the proportion of individuals with a twofold or greater increase from BL in specific IgG against two or more serotypes at 5 years. We also analyzed durable antibody response in both groups in terms of seroprotection rate at T5, defined as the proportion of responders for each serotype with an IgG level $\geq 0.35 \mu \mathrm{g} / \mathrm{ml}$ (accepted by the WHO for assessment of vaccine efficacy against IPD) [13] and $\geq 1 \mu \mathrm{g} / \mathrm{ml}$ (potentially more relevant for long-term protection and previously used in studies with HIV-infected children) [14] and by using the novel individual serotype-specific correlate of protection (established for all serotypes, except for serotype 5) [15]. The proportion of participants who achieved seroprotection to the majority of serogroups $(\geq 9)$ based on the three cutoffs was also evaluated.

In the statistical analyses, the resulting $p$ values were corrected for multiple testing by controlling the false discovery rate (FDR) to $\leq 0.05$ through the Benjamini-Hochberg method. Analyses were performed using the SPSS software package (version 22.0, Chicago, IL, USA).

\section{RESULTS}

We analyzed 91 subjects (out of the 100 patients analyzed in the previous report) [11]: $72.5 \%$ males; median age 50 years (IQR 45-56); median $\mathrm{CD}^{+} 650$ cells/ $\mu \mathrm{l}$ (IQR 506-881). All patients were on antiretroviral therapy, $>90 \%$ with HIV-RNA $<50$ copies $/ \mathrm{ml}$; patients in groups $1(n=42)$ and $2(n=49)$ were homogeneous for the main characteristics and were comparable to the entire original study population [11] (Table 1).

Pre-vaccination serotype-specific IgG levels were comparable between the two groups, in agreement with the original cohort (all ps n.s.) [11]. The long-term serologic response was markedly higher for serotype $19 \mathrm{~F}$ in the group immunized with PCV13 compared with the PPV23 group; the specific IgG GMCs for all other pneumococcal antigens did not differ between groups (Table 2). 
Table 1 Characteristics of HIV-infected patients who received either two doses of PCV13 (group 1) or one dose of PPV23 (group 2) 5 years after vaccination

\begin{tabular}{|c|c|c|c|}
\hline & $\begin{array}{l}\text { Group 1 } \\
\text { (PCV13) } \\
n=42\end{array}$ & $\begin{array}{l}\text { Group 2 } \\
\text { (PPV23) } \\
n=49\end{array}$ & $p$ \\
\hline Male, $n(\%)$ & $28(66.7)$ & $38(77.6)$ & 0.246 \\
\hline Age, mean $(\mathrm{SD})$ & $49.5(9.5)$ & $50.3(10.6)$ & 0.685 \\
\hline Non-Caucasian, $n(\%)$ & $5(11.9)$ & $1(2.0)$ & 0.091 \\
\hline $\begin{array}{l}\text { Body mass index }(\mathrm{kg} / \\
\left.\mathrm{m}^{2}\right) \text {, mean }(\mathrm{SD})\end{array}$ & $24.2(4.3)$ & $23.3(3.6)$ & 0.295 \\
\hline $\begin{array}{l}\text { Years from HIV } \\
\text { diagnosis, mean (SD) }\end{array}$ & $15.2(7.07)$ & $16.1(7.8)$ & 0.553 \\
\hline $\begin{array}{l}\text { Years from first } \\
\text { HAART, mean (SD) }\end{array}$ & $13.3(7.09)$ & $13.1(5.9)$ & 0.844 \\
\hline Risk factor, $n$ (\%) & & & 0.354 \\
\hline Heterosexual & $14(33.3)$ & $20(40.8)$ & \\
\hline Homo-/bisexual & $15(35.7)$ & $20(40.8)$ & \\
\hline Injection drug use & $7(16.7)$ & $7(14.3)$ & \\
\hline Other/unknown & $6(14.3)$ & $2(4.1)$ & \\
\hline $\begin{array}{l}\text { Nadir CD4 count (cell/ } \\
\mu \mathrm{l}), \text { mean }(\mathrm{SD})\end{array}$ & $189(130)$ & $213(192)$ & 0.492 \\
\hline $\begin{array}{l}\text { Current CD4 count } \\
(\text { cell } / \mu \mathrm{l}) \text {, mean }(\mathrm{SD})\end{array}$ & $708(249)$ & $689(295)$ & 0.744 \\
\hline \multicolumn{3}{|c|}{ Current CD 4 cell count, $n(\%)$} & 0.693 \\
\hline $200-350$ & $5(11.9)$ & $4(8.3)$ & \\
\hline $351-500$ & $7(16.7)$ & $6(12.5)$ & \\
\hline$>500$ & $30(71.4)$ & $38(79.2)$ & \\
\hline $\begin{array}{l}\text { Pre-HAART HIV- } \\
\text { RNA (log HIV-RNA } \\
\text { copies/ml, mean } \\
\text { (SD) }\end{array}$ & $4.8(0.77)$ & $4.8(0.98)$ & 0.791 \\
\hline $\begin{array}{l}\text { Viral load } \leq 50 \text { copies/ } \\
\quad \mathrm{ml}, n(\%)\end{array}$ & $38(90.5)$ & $45(91.8)$ & 0.843 \\
\hline $\begin{array}{l}\text { Receipt of HAART, } \\
\quad n(\%)\end{array}$ & $42(100)$ & $49(100)$ & 1.000 \\
\hline \multicolumn{4}{|l|}{ Comorbidities, $n(\%)$} \\
\hline Diabetes & $2(4.8)$ & $0(0)$ & 0.210 \\
\hline
\end{tabular}

Table 1 continued

\begin{tabular}{|c|c|c|c|}
\hline & $\begin{array}{l}\text { Group 1 } \\
\text { (PCV13) } \\
n=42\end{array}$ & $\begin{array}{l}\text { Group 2 } \\
\text { (PPV23) } \\
n=49\end{array}$ & $p$ \\
\hline Chronic heart disease & $3(7.1)$ & $5(10.2)$ & 0.721 \\
\hline Neoplasm & $1(2.4)$ & $2(4.1)$ & 0.651 \\
\hline HCV co-infection & $6(14.2)$ & $6(12.2)$ & 0.834 \\
\hline $\begin{array}{l}\text { Recent hospitalization, } \\
n \text { (\%) (last } \\
12 \text { months) }\end{array}$ & $4(9.5)$ & $3(6.1)$ & 0.544 \\
\hline $\begin{array}{l}\text { Past AIDS-defining } \\
\text { events, } n(\%)\end{array}$ & $9(21.4)$ & $18(36.7)$ & 0.111 \\
\hline Smokers*, $n(\%)$ & $25(59.5)$ & $33(67.3)$ & 0.439 \\
\hline $\begin{array}{l}\text { Alcohol consumers**, } \\
n(\%)\end{array}$ & $6(14.3)$ & $4(8.2)$ & 0.504 \\
\hline
\end{tabular}

Comparing $\mathrm{BL}$ and $\mathrm{T} 5$, responses to serotypes $6 \mathrm{~B}, 19 \mathrm{~F}$ and $23 \mathrm{~F}$ were markedly higher in group 1 , whereas two antigens, i.e., 1 and 4 , elicited higher responses in group 2. The IgG titers for serotypes $5,7 \mathrm{~F}, 9 \mathrm{~V}, 14$ and $18 \mathrm{C}$ were significantly higher in patients immunized with both vaccines; antigens 3 and 19A did not show any variation (Table 3a, b).

With respect to T1, at T5 IgG titers for 4, 5, $6 \mathrm{~B}, 9 \mathrm{~V}, 14$ and $18 \mathrm{C}$ showed a statistically significant reduction in both groups; regarding antigens $19 \mathrm{~A}$ and $23 \mathrm{~F}$, the decay was greater in group 2 than group 1 . IgG titers $7 \mathrm{~F}$ and $19 \mathrm{~F}$ diminished only in group 2 , whereas serotypes 1 and 3 were stable in both groups (Table $3 a, b$ ). Analyzing the main effect of vaccine over time, $19 \mathrm{~F}$ was statistically significantly higher in PCV13 than in PPV23, although this effect appeared to be less marked when considering the contrast for interaction time-vaccine (Supplementary Table 1 ).

When considering the seroconversion rate, defined as $\mathrm{a} \geq$ twofold rise from baseline antibody levels at T5, a higher proportion of responders to the $6 \mathrm{~B}$ serotype were observed in the PCV13 group than in the PPV23 group, although it was not significant after adjusting 
Table 2 Comparison of long-term geometric mean concentrations (GMCs) of IgGs against the 12 serotypes between two groups of HIV-infected patients immunized with either PCV13 or PPV23 5 years earlier

\begin{tabular}{|c|c|c|c|c|c|c|c|c|c|c|}
\hline \multirow[t]{2}{*}{ Serotype } & \multirow[t]{2}{*}{ PCV13 GMC } & \multicolumn{2}{|c|}{ 95\% CI } & \multirow[t]{2}{*}{ PPV23 GMC } & \multicolumn{2}{|c|}{$95 \% \mathrm{CI}$} & \multirow[t]{2}{*}{ GM ratio ${ }^{a}$} & \multicolumn{2}{|c|}{$95 \% \mathrm{CI}$} & \multirow[t]{2}{*}{$p$} \\
\hline & & Lower & Upper & & Lower & Upper & & Lower & Upper & \\
\hline 1 & 2.57 & 2.04 & 3.24 & 2.82 & 2.29 & 3.47 & 1.11 & 0.82 & 1.50 & 0.481 \\
\hline 3 & 0.78 & 0.63 & 0.98 & 0.72 & 0.60 & 0.89 & 0.95 & 0.71 & 1.28 & 0.758 \\
\hline 4 & 0.98 & 0.76 & 1.26 & 0.93 & 0.74 & 1.15 & 1.00 & 0.71 & 1.41 & 0.992 \\
\hline 5 & 1.29 & 0.93 & 1.74 & 1.15 & 0.85 & 1.55 & 0.93 & 0.60 & 1.44 & 0.730 \\
\hline $6 \mathrm{~B}$ & 2.57 & 2.00 & 3.31 & 1.74 & 1.35 & 2.24 & 0.72 & 0.50 & 1.03 & 0.069 \\
\hline $7 F$ & 1.45 & 1.12 & 1.86 & 1.26 & 0.95 & 1.70 & 0.91 & 1.61 & 1.33 & 0.616 \\
\hline $9 \mathrm{~V}$ & 0.87 & 0.66 & 1.12 & 1.10 & 0.83 & 1.48 & 1.34 & 0.90 & 2.00 & 0.149 \\
\hline 14 & 9.12 & 6.76 & 12.30 & 11.48 & 8.71 & 15.14 & 1.28 & 0.86 & 1.91 & 0.223 \\
\hline $18 \mathrm{C}$ & 1.91 & 1.45 & 2.45 & 1.48 & 1.12 & 2.00 & 0.79 & 1.80 & 0.53 & 0.074 \\
\hline $19 \mathrm{~A}$ & 4.79 & 3.72 & 6.17 & 4.07 & 3.16 & 5.13 & 0.88 & 0.62 & 1.24 & 0.456 \\
\hline $19 \mathrm{~F}$ & 5.89 & 4.79 & 7.41 & 3.72 & 3.09 & 4.47 & 0.64 & 0.47 & 0.85 & 0.003 \\
\hline $23 \mathrm{~F}$ & 2.57 & 1.95 & 3.24 & 1.86 & 1.45 & 2.95 & 0.75 & 0.52 & 1.08 & 0.124 \\
\hline
\end{tabular}

${ }^{a}$ Multivariable linear regression was adjusted with age, gender and CD4 cell counts

Significant $p$ values are in bold

Significance was calculated from $p$ values using the Benjamini-Hochberg false discovery rate test $(\leq 0.05)$

for FDR; for the remaining serotypes, proportions of responders were comparable (Supplementary Table 2). Notably, considering the percentage of responders designed by a twofold or greater increase in antibody levels to two or more serotypes, more participants in the PCV13 group had a persistent antibody response than those in the PPV23 group at 5 years (78.6 vs. $59.2 \%$, AOR $0.37 \quad 95 \%$ CI $0.144 / 0.966$, $p=0.042)$. The seroprotection rate at $\mathrm{T} 5$ was similar for all 12 serotypes between PCV13 and PPV23 based on a threshold $\geq 0.35, \geq 1 \mu \mathrm{g} / \mathrm{ml}$ and considering serotype-specific clinically validated thresholds (correlates of protection) (Supplementary Table $3 \mathrm{~A}, \mathrm{~B}$ and C). In both the PCV13 and PPV23 group, most of the participants achieved seroprotection to the majority of serogroups ( $\geq 9$ ) based on IgG levels $\geq 0.35 \mu \mathrm{g}$ / $\mathrm{ml}$ (97.6 vs. 98.0\%, AOR 1.47 95\% CI $0.082 /$ $26.32, p=0.794), \geq 1 \mu \mathrm{g} / \mathrm{ml}$ (54.8 vs. $49.0 \%$, AOR $0.8995 \%$ CI $0.38 / 2.11, p=0.792$ ) and $\geq$ serotype-specific clinically validated thresholds (88.1 vs. $75.5 \%$, AOR $0.4195 \%$ CI $0.13 / 1.31, p=0.134)$.

During the 5 years from the first vaccination, no invasive pneumococcal disease occurred in any patient; HIV-RNA level and $\mathrm{CD}^{+}$cells counts remained stable over time.

\section{DISCUSSION}

The aim of this report was to compare the longterm response according to IgG levels in a study population that included HIV-positive adults who had received either one dose of PPV23 or two doses of PCV13 5 years earlier.

Previously we reported that in the same cohort of patients with a favorable viro-immunologic profile, PCV13 and PPV23 showed comparable immune-specific IgG responses to the 12 shared pneumococcal antigens at a 1-year follow-up [11].

In this report, which compared the IgG levels of the two groups 5 years after vaccination, one 


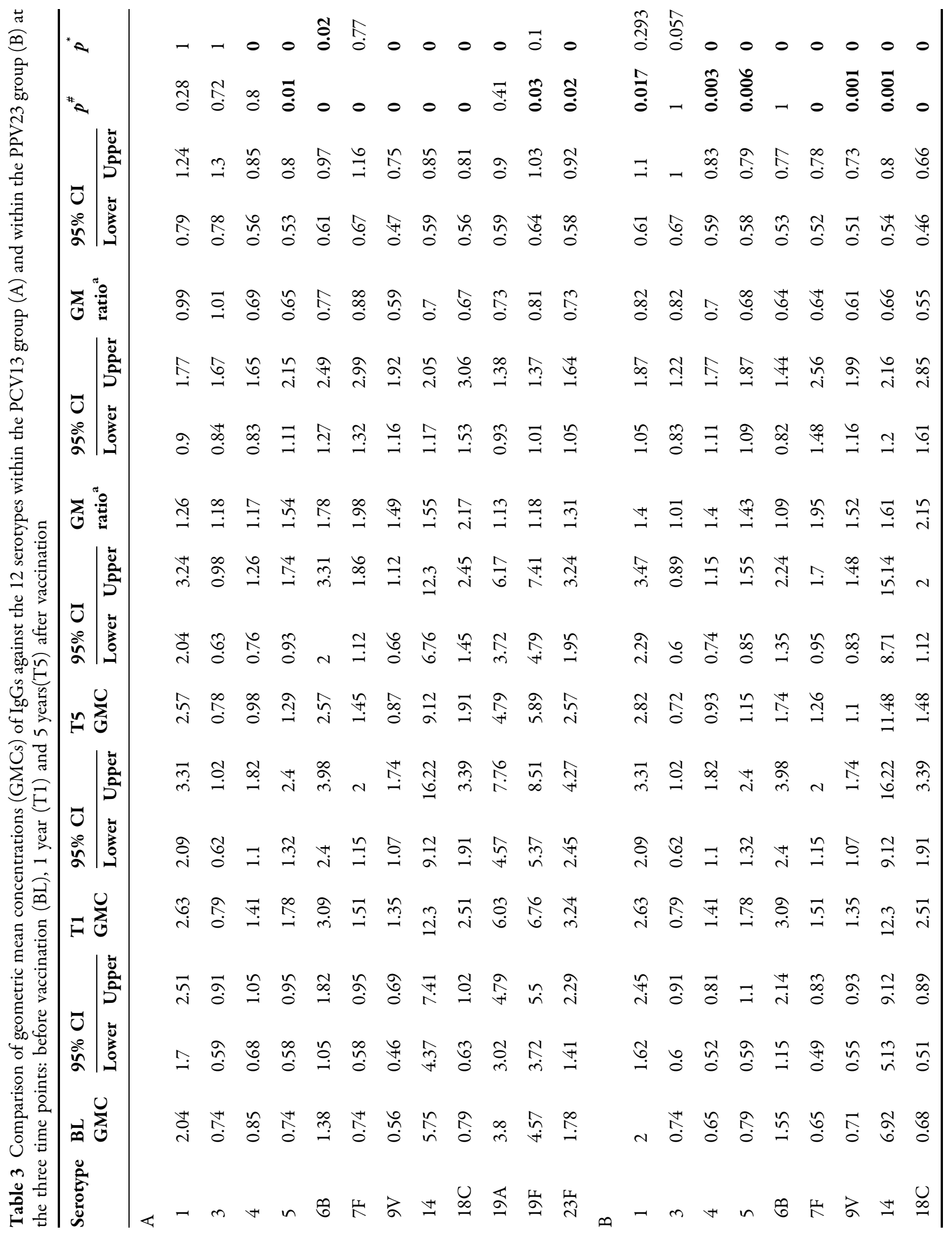




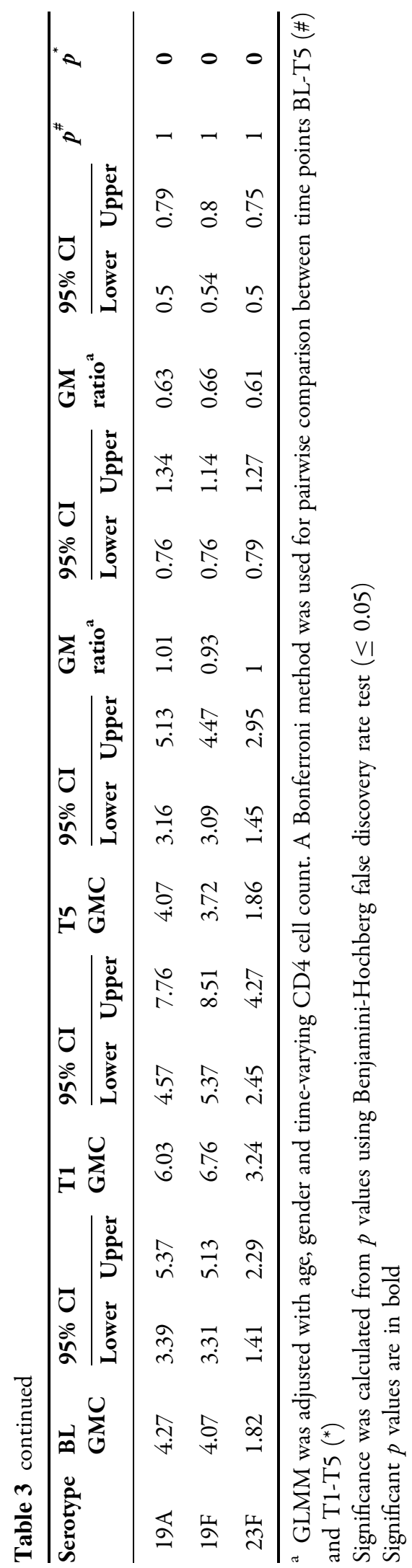

antigen, i.e., 19F, was significantly higher in the PCV13 group. When T5 was compared with BL, more antigens displayed higher GMCs levels in the PCV13 than in the PPV23 group, i.e., 8 versus 7, respectively. With respect to 1 year, GMCs decreased significantly at $\mathrm{T} 5$ in the PCV13 group for eight serotypes versus ten serotypes in the PPV23 group. In terms of subjects with a twofold or greater increase in specific IgG against two or more serotypes, the PCV13 group showed a greater proportion of responders.

Although the differences in long-term immunogenicity between PCV13 and PPV23 were not drastically relevant, these combined results suggest that in the long-term the development of memory cells via a T-cell-dependent response elicited by PCV13 could be an advantage in terms of antibody persistence compared with PPV23 in HIV-positive adults. This higher level of antibody persistence is a novel finding in this population. In a previous analysis, Cheng et al. [10] documented a durable antibody response in HIV-positive adults 5 years after vaccination with PCV7; however, in that study a group of subjects receiving PPV was lacking for comparison.

Overall, both vaccines seem to have achieved a durable serologic response 5 years after immunization. Indeed, PCV13 and PPV23 revealed (1) significantly higher GMCs than BL to most serotypes, (2) a $\geq$ twofold increase in antibody levels to $\geq$ two serotypes in $79 \%$ and $59 \%$ of patients, respectively, and (3) the achievement of seroprotection (based on the defined thresholds) to the majority of the 12 serogroups in a large percentage of participants.

Regarding the response to PPV23, our findings appear to be in contrast to previous reports, which showed that the immunogenicity and durability of 23-valent PPV are poor in HIV-infected patients $[9,16,17]$; this probably suggests the effect of the different definitions of serologic response, number of serotypes examined and study population with a longer follow-up on antiretroviral therapy or different viro-immunologic statuses. Of note, our population mainly consisted of virologically suppressed HIV-infected patients who had good immunologic recovery; this is important because the 
$\mathrm{CD}^{+}$cell count has been shown to be a favorable predictor of the response rate for patients who have received a PPV23 vaccination $[9,18,19]$.

Consistent with previous data that showed a lower immunogenicity of this serotype, antigen 3 did not elicit a significant long-term immunologic response in either group [20]. In our study, the two-dose schedule for PCV13 was designed when there was no defined standard regimen recommended for use of PCV 13 in HIV-positive adults (year 2011), and the need for a boosted dose was assumed based on previous data obtained in children [21]. Currently, even if the guidelines recommend one dose of PCV13 and there are no general recommendations for booster doses, some national guidelines consider that one dose of PPV23 should be administered at least 2 months after PCV13 [5]. However, there is still no definite consensus. Our scheduled PCV13 booster could represent a limit in interpreting our results in actual clinical practice, even though we previously reported no significant increase in IgG GMCs after the second dose of PCV13 compared with after the first dose [11], in line with other studies [22, 23]. Sequential vaccination with PCV13 followed by PPV23 to boost the antibody secretion of common serotypes and additional data regarding long-term immunization effects of the serotypes unique to PCV13 should be carried out in ongoing/future studies, which could be compared with our results. Our study has several limitations due to the relatively small sample size and the absence of an a priori design as a randomized trial. However, at the T5 time point, the two groups were comparable for demographic, clinical and viro-immunologic parameters, as consistently reported at the time of vaccination; moreover, at that time serotypespecific IgG concentrations were similar between groups. In addition, we performed analyses adjusting for potential confounders that could affect the comparison of long-term effects of the two vaccines. Another limitation is that, because of the small sample size, we were unable to compare clinical outcomes of infections according to vaccine group.

The main strength of the study is the assessment of all 12 serotypes shared by both
PCV13 and PPV23. However, further investigations are needed in HIV-infected adults with different viro-immunologic profiles.

\section{CONCLUSION}

Finally, to the best of our knowledge, this study is the first direct comparison of long-term serologic response of the conjugated vs. polysaccharide vaccine in HIV-positive adults.

In our study overall both vaccines were shown to achieve a long-term durable serologic response. Nevertheless, we found minor differences in immunogenicity between the two vaccines, which favored PCV13 over PPV23 5 years after immunization as PCV13 elicited significantly higher GMC levels than the $19 \mathrm{~F}$ serotype, higher GMCs for a greater number of antigens when compared with baseline values, a significant decrease in GMCs for fewer antigens with respect to T1 levels and a higher proportion of patients who had $\mathrm{a} \geq$ twofold increase in antibody titers from baseline to two or more serotypes.

\section{ACKNOWLEDGMENTS}

The authors would like to thank the clinical staff and the participants of the study.

Funding. No funding or sponsorship was received for this study or publication of this article. The Rapid Service Fees were funded by the authors.

Authorship. All named authors meet the International Committee of Medical Journal Editors (ICMJE) criteria for authorship for this article, take responsibility for the integrity of the work as a whole, and have given their approval for this version to be published.

Disclosures. Barbara Rossetti received a grant for educational activities from Abbvie, Gilead Sciences, Janssen-Cilag, Merck Sharp and Dohme, ViiV healthcare and Bristol-Myers Squibb and personal fees for advisory boards and speakers bureaus from Abbvie, Gilead 
Sciences, Janssen-Cilag, Merck Sharp and Dohme, ViiV healthcare and Bristol-Myers Squibb. Alberto Borghetti has received non-financial support from Bristol-Myers Squibb and ViiV Healthcare and personal fees from Gilead Sciences. Roberto Cauda has been an advisor for Gilead, Janssen-Cilag and Basel Pharmaceutical, has received speakers' honoraria from ViiV Healthcare, Bristol-Myers Squibb, Merck Sharp \& Dohme, Abbott, Gilead and Janssen-Cilag, and has received research support from 'Fondazione Roma.' Andrea De Luca received research grants from MSD, ViiV and Gilead Sciences and consultant fees from AbbVie, BMS, Gilead, MSD, ViiV and Janssen-Cilag. Francesca Montagnani reported non-financial support from Angelini, outside the submitted work: she has done contract research for Novartis Vaccine and Diagnostic S.rl. (now GSK Vaccine S.r.l.) on behalf of the University Hospital of Siena. She is Infectious Diseases Consultant for GSK (consultancy fee on behalf of University of Siena). Simone Belmonti, Sara Modica, Lorenzo Paglicci, Arturo Ciccullo, Chiara Picarelli and Francesca Lombardi have nothing to disclose.

Compliance with Ethics Guidelines. All procedures performed in the study were in accordance with the 1964 Helsinki Declaration and its later amendments or comparable ethical standards. The present study was approved by the local Institutional Ethics Committees, Comitato Etico Fondazione Policlinico universitario "Agostino Gemelli"- Università Cattolica del Sacro Cuore, and Comitato Etico Regione Toscana-Area vasta Sud Est, AOU Senese (protocol no. 13379/16 and protocol code PCV13HIV-BOOST-2014). Written informed consent was obtained from each participant.

Data Availability. The datasets generated during and/or analyzed during the current study are available from the corresponding author on reasonable request.

Open Access. This article is distributed under the terms of the Creative Commons Attribution-NonCommercial 4.0 International License (http://creativecommons.org/licenses/ by-nc/4.0/), which permits any noncommercial use, distribution, and reproduction in any medium, provided you give appropriate credit to the original author(s) and the source, provide a link to the Creative Commons license, and indicate if changes were made.

\section{REFERENCES}

1. Jordano $\mathrm{Q}$, Falco $\mathrm{V}$, Almirante $\mathrm{B}$, et al. Invasive pneumococcal disease in patients infected with HIV: still a threat in the era of highly active antiretroviral therapy. Clin Infect Dis. 2004;38(11):1623-8.

2. Hart M, Steel A, Clark SA, et al. Loss of discrete memory B cell subsets is associated with impaired immunization responses in HIV-1 infection and may be a risk factor for invasive pneumococcal disease. J Immunol. 2014;178(12):8212-20.

3. Iwajomo OH, Finn A, Ogunniyi AD, Williams NA, Heyderman RS. Impairment of pneumococcal antigen specific isotype-switched Igg memory B-cell immunity in HIV infected Malawian adults. PLoS One. 2013;8(11):e78592.

4. French N, Gordon SB, Mwalukomo T, et al. A trial of a 7-valent pneumococcal conjugate vaccine in HIVinfected adults. N Engl J Med. 2010;362(9):812-22.

5. Tomczyk S, Bennett NM, Stoecker C, et al. Use of 13-valent pneumococcal conjugate vaccine and 23-valent pneumococcal polysaccharide vaccine among adults aged $\geq 65$ years: recommendations of the Advisory Committee on Immunization Practices (ACIP). MMWR Morb Mortal Wkly Rep. 2014;63(37):822-5.

6. Ho YL, Brandao AP, De Cunto Brandileone MC, et al. Immunogenicity and safety of pneumococcal conjugate polysaccharide and free polysaccharide vaccines alone or combined in HIV-infected adults in Brazil. Vaccine. 2013;31(37):4047-53.

7. Feikin DR, Elie CM, Goetz MB, et al. Randomized trial of the quantitative and functional antibody responses to a 7-valent pneumococcal conjugate vaccine and/or 23-valent polysaccharide vaccine among HIV-infected adults. Vaccine. 2001;20(3-4):545-53.

8. Lesprit P, Pedrono G, Molina JM, et al. Immunological efficacy of a prime-boost pneumococcal vaccination in HIV-infected adults AIDS. 2007;21(18):2425-34. 
9. Hung CC, Chang SY, Su CT, et al. A 5-year longitudinal follow-up study of serological responses to 23-valent pneumococcal polysaccharide vaccination among patients with HIV infection who received highly active antiretroviral therapy. HIV Med. 2010;11(1):54-63.

10. Cheng A, Sui-Yuan C, Mao-Song T, et al. Long-term immune responses and comparative effectiveness of one or two doses of 7-valent pneumococcal conjugate vaccine (PCV7) in HIV-positive adults in the era of combination antiretroviral therapy. J Int AIDS Soc. 2016;19(1):20631.

11. Lombardi F, Belmonti S, Fabbiani $M$, et al. Immunogenicity and safety of the 13-valent pneumococcal conjugate vaccine versus the 23 -valent polysaccharide vaccine in unvaccinated hiv-infected adults: a pilot, prospective controlled study. PLoS One. 2016;11(6):e0156523.

12. Belmonti S, Lombardi F, Morandi M, et al. Evaluation and optimization of an ELISA procedure to quantify antibodies against pneumococcal polysaccharides included in the 13-valent conjugate vaccine. $\mathrm{J}$ Immunoassay Immunochem. 2016;37(2):189-200.

13. World Health Organization. Recommendation for the production and control of pneumococcal conjugate vaccines. WHO Technical Report Series. No. 927, 2005.

14. Abzug MJ, Pelton SI, Song LY, et al. Immunogenicity, safety, and predictors of response after a pneumococcal conjugate and pneumococcal polysaccharide vaccine series in human immunodeficiency virus-infected children receiving highly active antiretroviral therapy. Pediatr Infect Dis J. 2006;25(10):920-9 Epub 2006/09/29.

15. Andrews NJ, Waight PA, Burbidge P, et al. Serotypespecific effectiveness and correlates of protection for the 13-valent pneumococcal conjugate vaccine: a postlicensure indirect cohort study. Lancet Infect Dis. 2014;14(9):839-46.
16. Kroon FP, Van Dissel JT, Ravensbergen E, et al. Enhanced antibody response to pneumococcal polysaccharide vaccine after prior immunization with conjugate pneumococcal vaccine in HIV-infected adults. Vaccine. 2000;19(7-8):886-94.

17. French N, Gilks CF, Mujugira A, et al. Pneumococcal vaccination in HIV-1 infected in Uganda: humoral response and two vaccine failures. AIDS. 1998;12(13):1683-9.

18. French N, Nakiyingi J, Carpenter LM, et al. 23-valent pneumococcal polysaccharide vaccine in HIV-1 infected Ugandian adults: double-blind, randomised and placebo controlled trial. Lancet. 2000;355(9221):2106-11.

19. Rodriguez-Barradas MC, Alexandraki I, Nazir T, et al. Response of human immunodeficiency virusinfected patients receiving highly active antiretroviral therapy to vaccination with 23 -valent pneumococcal polysaccharide vaccine. Clin Infect Dis. 2003;37(3):438-47.

20. Munoz-Almagro C, Navarro-Torne A, Pallares R, et al. Epidemiologic and clinical implications of second-generation pneumococcal conjugate vaccines. Curr Infect Dis Rep. 2013;15(2):184-90.

21. Bliss SJ, O'Brien KL, Janoff EN, et al. The evidence for using conjugate vaccines to protect HIV-infected children against pneumococcal disease. Lancet Infect Dis. 2008;8:67-80.

22. Glesby MJ, Watson W, Brinson C, et al. Immunogenicity and safety of 13-valent pneumococcal conjugate vaccine in HIV-infected adults previously vaccinated with pneumococcal polysaccharide vaccine. J Infect Dis. 2015;212(1):18-27 Epub 2014/11/15.

23. Bhorat AE, Madhi SA, Laudat F, et al. Immunogenicity and safety of the 13-valent pneumococcal conjugate vaccine in HIV-infected individuals naive to pneumococcal vaccination. AIDS. 2015;29(11): 1345-54 (Epub 2015/04/19). 